\title{
Enjeux de la biologie délocalisée en milieu hospitalier et contractualisation
}

\author{
J.-P. Borgard \\ Coordonnateur de la section biologie \\ délocalisée du Collège National de \\ Biochimie des Hôpitaux. \\ jp.borgard@chicreteil.fr
}

Laboratoire de Biochimie ; Centre Hospitalier Intercommunal de Créteil ,40 avenue de Verdun 94010 Créteil

\section{INTRODUCTION}

Est-il "biologiquement correct" de considérer la délocalisation de certains actes réalisés dans le cadre des laboratoires de biologie comme une amélioration de la prise en charge du patient?

Les plus âgés d'entre nous ont connu l'existence des laboratoires de service. L'évolution technologique et l'automatisation ont depuis conduit à une centralisation des moyens et les progrès de l'informatique en terme de circulation d'information ont définitivement rendu ce mode de fonctionnement obsolète.

Les services de biologie se sont investis dans des activités transversales et sont actueliement, dans bon nombre d'hôpitaux, considérés comme un pôle structurant du volet "médical" des projets d'établissement. A ce titre, ils sont amenés à réfléchir, en coopération avec les services de soins et les services économiques aux moyens matériels et humains à mettre en œuvre pour améliorer l'efficacité de la prise en charge du patient. Aujourd'hui, la réalisation hors les murs des laboratoires institutionnels d'analyses de biologie médicale peut représenter, dans des situations bien ciblées et limitées, une solution intellectuellement recevable. Pour autant, la délocalisation d'analyses biologiques ne peut être $n$ anarchique ni systématique. Elle doit répondre à des critères et suivre des règles d'implantation ayant fait l'objet de consensus et finalisées par l'établissement de contrats entre les services cliniques, biologiques et administratifs.

\section{CRITERES DE DELOCALISATION}

Une demande de délocalisation doit, non seulement avoir reçu l'aval des soignants, mais aussi s'inscrire dans un cadre biologiquement et économiquement acceptable.

Pour le biologiste,

- les résultats obtenus en délocalisé doivent avoir une fiabilité identique à ceux du laboratoire,

- la délocalisation doit améliorer d'une manière significative le mode de fonctionnement pré-existant, - le processus mis en place doit respecter des règles d'assurance qualité et du Guide de Bonne Exécution des Analyses (GBEA). Pour les services économiques les améliorations escomptées doivent être suffisamment convaincantes et patentes pour justifier un surcoût qu'il est nécessaire de chiffrer précisèment

C'est le cadre dans lequel doivent se dérouler les discussions entre les différents acteurs.

\section{LES DIFFERENTES ETAPES}

\section{Etude de la pertinence}

L'obtention plus rapide du résultat et la prise en charge thérapeutique plus précoce sont les arguments les plus souvent invoqués. Les soignants ont tendance à considérer que l'acte analytique est très simple (machines "presse-bouton") et méconnaissent totalement les répercussions de la qualité du prélèvement sur la fiabilité du résultat. Une conséquence directe de la phase préparatoire d'une délocalisation est la prise de conscience de l'importance de la phase pré-analytique pour obtenir un résultat fiable et donc utilisable localement.

Les autres arguments varient en fonction du lieu de délocalisation envisagé : proximité (blocs opératoires, salle de réveil), connexion possible au système de monitorage voire embarquement dans les véhicules SAMU etc... A titre d'exemple, je présenterai les avantages escomptés d'une biologie délocalisée au chevet du patient dans le service de réanimation néonatale du Centre Hospitalier Intercommunal de Créteil. Le cahier des charges médical comprenait la réalisation d'analyses de gazométrie sanguine couplées à celle d'électrolytes (Sodium, Potassium, Calcium ionisé voire Chlore), de certains métabolites (urée, glucose), le plus près possible du patient, et sur un volume minimum de prélèvement capillaire.

Le faible volume de prélèvement et la possibilité d'effectuer l'analyse au moment pertinent permettaient d'espérer une épargne sanguine primordiale dans le cas d'une très grande prématurité (recrutement majeur du service).

Le prélèvement capillaire diminue le risque d'infection voire de nécrose locale inhérent aux prélèvements centraux chez des enfants au statut immunologique précaire et préserve le capital artériel et veineux. II diminue en plus la douleur de l'enfant et le stress du préleveur. La réalisation des analyses à la demande améliore les flux au laboratoire et dans le service de soins (plus d'examens 